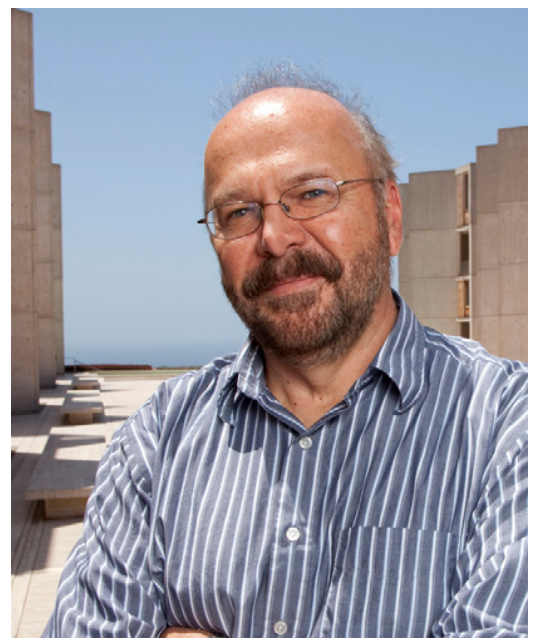

Joseph R. Ecker

\title{
The 2011 George W. Beadle Award
}

\author{
Joseph R. Ecker
}

$\mathrm{T}$

HE 2011 George W. Beadle Medal for outstanding contributions to the genetics community is awarded to Joseph R. Ecker. Joe is one of those rare scientists who has made fundamental discoveries in his own laboratory while also generating the genomic resources to enable discoveries in laboratories worldwide. Joe pioneered the use of genetics and genomics to dissect complex plant physiological processes. However, unlike most of us who are users of technology and databases developed elsewhere, Joe has consistently been ahead of his time and, as such, his is more often than not the laboratory where those nextgeneration technologies and data sets are developed.

Joe grew up in the mining town of Mount Carmel, Pennsylvania. He earned a BS in Biology in 1978 at the College of New Jersey where he discovered a passion for "doing science" as he sought out classes with laboratory sections. He earned a Ph.D. in Microbiology at Pennsylvania State University College of Medicine with virologist Richard Hyman. For his dissertation he used the cutting edge technology of the time, restriction enzymes and Southern blots, to characterize the genome of the chicken pox virus, varicella zoster (ECKER and HyMAN 1982).

Joe's move into plant biology was catalyzed by a National Science Foundation postdoctoral fellowship award designed specifically to attract those with training in molecular biology to switch their focus to plant systems. He joined the laboratory of Ron Davis in the Department of Biochemistry at Stanford University who was also broadening his focus from yeast to plants. Joe began his studies on the mechanism of action of ethylene using carrot cell culture where the hormone could be rapidly induced in 5 to $10 \mathrm{~min}$. The lack of a genetic handle eventually prompted him to adopt the emerging genetic plant model Arabidopsis thaliana. However, before doing so, he used carrot protoplasts to demonstrate for the first time that antisense RNA could inhibit gene expression in a plant (ECKER and DAvis 1986).
With a collection of mutagenized Arabidopsis seeds in tow, Joe joined the faculty of the University of Pennsylvania in 1987 and quickly discovered that isolating ethylene response mutants was not going to be a limiting factor in his research. Instead, he had to devise strategies to begin to isolate the numerous genes and figure out how their products interacted to produce the spectrum of ethylene responses. This quest, which continues to this day, led to the earliest mechanistic understanding of a plant hormone. Perhaps more importantly, Joe has made the resources developed in his laboratory over the years freely available and, in this way, has fueled the growth of the Arabidopsis community, as young scientists seized the opportunity to make fundamental discoveries in the plant kingdom.

In a series of landmark studies beginning at the University of Pennsylvania and continuing at the Salk Institute (where he is professor in the Plant Biology Laboratory and director of the Salk Institute Genomic Analysis Laboratory), Joe's laboratory developed the resources and methodologies necessary for the Arabidopsis community to move from mutants to molecules. First with methodologies for isolating large DNA fragments, thus enabling construction of the first YAC and BAC libraries of a plant genome (GUZMAN and ECKER 1988), next with the mapping of the first plant microsatellite markers (BELL and Ecker 1994), and then with Sakis Theologis and Ron Davis, Joe led the charge to sequence the Arabidopsis genome which, when completed ahead of schedule in 2000, was only the third high-quality finished eukaryotic genome (ARABIDOPSIS Genome Initiative 2000).

Before the genome sequence was even complete, Joe's laboratory was developing tools that would be needed to make it more biologically meaningful. To verify, correct, and extend genome annotation Joe (in collaboration with Sakis Theologis) led a group that generated and sequenced full-length cDNA sequences and hybridized RNAs to whole genome tiling arrays thus 
identifying almost 6000 novel transcription units (YAMAda et al. 2003). This feat was matched by what is arguably Joe's most significant contribution to plant biology: the enormous collection of T-DNA insertion lines. One measure of the impact of this resource is that the publication reporting the 225,000 independent insertions; 88,000 of which were precisely located (Alonso et al. 2003), has been cited over 2100 times to date. The "Salk Collection," as it is now known, allows researchers worldwide to use e-mail to order mutants of interest in almost any Arabidopsis gene.

Over the past 5 years, Joe has turned his attention to the epigenome, leading his laboratory in the development of the first whole-genome high-resolution map of DNA methylation. This study (in collaboration with Steve Jacobsen) led to the surprising discovery of methylation within transcribed genes. This feature of the epigenome, now called gene body methylation, has subsequently been found in animals including human (ZHANG et al. 2006). Recently, they have exploited high throughput DNA sequencing to refine the methylome to single-base resolution, first in Arabidopsis (Lister et al. 2008) and then in human stem cells (Lister et al. 2009).

Joe has also effectively served the plant community as an ambassador to the international scientific community. As a member of numerous government advisory boards, he has effectively lobbied for funds by keeping plant research on their radar. He has been a key participant in several long-term planning studies for the National Science Foundation and has held leadership positions in several plant associations including service as president of the International Society of Plant Molecular Biology (2005-2008). Over the years, he has been recognized by several honors for both his scholarship and community service, including the American Society for Plant Molecular Biology Martin Gibbs Medal (2005), election to the National Academy of Sciences (2006), and the National Academy of Sciences John J. Carty Award for the Advancement of Science (2007).
Given his enormous contributions to plant biology, it should be noted that Joe never took a plant biology course as an undergraduate. As mentioned above, he took courses with laboratory sections and no plant laboratories were available. Fittingly, the very resources that he is being recognized with this award for generating in Arabidopsis-from the genome sequence to the indexed T-DNA insertions, to the whole genome chips to the methylome-are preparing the next generation of plant scientists by making it possible for undergraduates to investigate plant genomes side by side with their animal counterparts.

\section{LITERATURE CITED}

Arabidopsis Genome Iniative, 2000 Analysis of the genome sequence of the flowering plant Arabidopsis thaliana. Nature 408: 796-815.

Alonso, J. M., A. N. Stepanova, T. J. Leisse, C. J. Kim, H. Chen et al., 2003 Genome-wide insertional mutagenesis of Arabidopsis thaliana. Science 301: 653-657.

Bell, C. J., and J. R. Ecker, 1994 Assignment of 30 microsatellite loci to the linkage map of Arabidopsis. Genomics 19: 137-144.

ECKER, J. R., and R. W. DAvis, 1986 Inhibition of gene expression in plant cells by expression of antisense RNA. Proc. Natl. Acad. Sci. USA 83: 5372-5376.

Ecker, J. R., and R. W. Hyman, 1982 Varicella zoster virus DNA exists as two isomers. Proc. Natl. Acad. Sci. USA 79: 156-160.

Guzman, P., and J. R. Ecker, 1988 Development of large DNA methods for plants: molecular cloning of large segments of Arabidopsis and carrot DNA into yeast. Nucleic Acids Res. 16: 11091-11105.

Lister, R., R. C. O’Malley, J. Tonti-Filippini, B. D. Gregory, C. C. BERry et al., 2008 Highly integrated single-base resolution maps of the epigenome in Arabidopsis. Cell 133: 523-536.

Lister, R., M. Pelizzola, R. H. Dowen, R. D. Hawkins, G. Hon et al., 2009 Human DNA methylomes at base resolution show widespread epigenomic differences. Nature 462: 315-322.

Yamada, K., J. Lim, J. M. Dale, H. Chen, P. Shinn et al., 2003 Empirical analysis of transcriptional activity in the Arabidopsis genome. Science 302: 842-846.

Zhang, X., J. Yazaki, A. Sundaresan, S. Cokus, S. W. Chan et al., 2006 Genome-wide high-resolution mapping and functional analysis of DNA methylation in arabidopsis. Cell 126: 1189-1201. 